\title{
A prospective study about impact of renal dysfunction and morbidity and mortality on cardiovascular events after ischemic stroke
}

\author{
Meta Penko ${ }^{1}$, Tanja Hojs Fabjan ${ }^{2}$, Sebastjan Bevc ${ }^{3}$, Vojko Kanič ${ }^{1}$, Radovan Hojs ${ }^{3}$ \\ ${ }^{1}$ Department of Cardiology, Clinic for Internal Medicine, University Medical Center, Maribor, Slovenia \\ ${ }^{2}$ Department of Neurology, University Medical Center, Maribor, Slovenia \\ ${ }^{3}$ Department of Nephrology, Clinic for Internal Medicine, \\ University Medical Center, Maribor, Slovenia
}

\begin{abstract}
Background: The aim of our prospective study was to define the impact of renal dysfunction on future cardiovascular events and total mortality in 390 patients suffering from ischemic stroke.
\end{abstract}

Methods: A quantitative measurement of neurologic deficit according to National Institutes of Health Stroke Scale (NIHSS) score was performed. Blood parameters were measured. Diabetes, hypertension and smoking habits were defined. Estimated glomerular filtration rate was calculated.

Results: 153 (39.2\%) patients had renal dysfunction. In the follow-up period in 36 (9.2\%) patients acute coronary syndrome, in 102 (26.2\%) recurrent ischemic stroke and in $44(11.3 \%)$ peripheral arterial disease were documented. 191 (49\%) patient died, 118 (30.3\%) of whom died of cardiovascular events. Patients who died were older, had higher prevalence of renal dysfunction and NIHSS score. The Kaplan-Meier survival analysis showed that total mortality $(p<0.003)$ and cardiovascular mortality $(p<0.01)$ were higher in patients with renal dysfunction. According to Cox's regression analysis, renal dysfunction was the predictor of cardiovascular events, cardiovascular and total mortality.

Conclusions: Patients with ischemic stroke and renal dysfunction are at higher risk for long term cardiovascular and total mortality. The patients with ischemic stroke and renal dysfunction are also at higher risk of new cardiovascular morbidity. Renal dysfunction should be added to the other known prognostic factors in patients with ischemic stroke. Our results also emphasize the importance of identification and management of renal dysfunction in stroke patients. (Cardiol J 2014; 21, 2: 163-169)

Key words: renal dysfunction, ischemic stroke, coronary heart disease, cardiovascular events, mortality

Address for correspondence: Meta Penko, MD, MSc, Univerzitetni klinični center Maribor, Klinika za interno medicino, Oddelek za kardiologijo in angiologijo, Ljubljanska 5, 2000 Maribor, Slovenia, tel: +386 2321 2871, fax: +386 23312393 , e-mail: meta.penko@gmail.com

Received: 10.04.2013 Accepted: 22.05.2013

These authors take responsibility for all aspects of the reliability and freedom from bias of the data presented and their discussed interpretation. 


\section{Introduction}

Coronary heart disease (CHD) and stroke are the main forms of the cardiovascular disease (CVD) which is the leading cause of death in Europe, United States and in many other developed countries [1]. Chronic kidney disease (CKD) is also a worldwide public health problem since the incidence and the prevalence is rising [2,3]. Patients with renal dysfunction have high prevalence of $\mathrm{CHD}$ and the prevalence increases with worsening renal function, reaching $40 \%$ in patients with end stage renal disease (ESRD) $[4,5]$. Mortality in ESRD patients due to CVD is from 10 to 20 times higher than in general population [6]. The main reason for high prevalence of CHD in patients with renal dysfunction is accelerated atherosclerosis, which starts already in early stages of renal failure [7]. Patients with earlier stages of CKD are not only at increased risk of $\mathrm{CHD}$ but have also a higher $\mathrm{CV}$ mortality compared to general population $[1,2,4,8]$. Furthermore, patients with CKD are more likely to die of CVD than to start with replacement therapy because of ESRD [9].

It was also shown that in patients with CHD the prevalence of renal dysfunction is higher than in general population and is estimated to be $18-37 \%$ $[8,10]$. Renal dysfunction was independently associated with an increased risk of death in patients with acute coronary syndrome (ACS) and was also strong and independent predictor of increased in-hospital and 1-year mortality after percutaneous coronary intervention $[8,10]$.

Patients with CKD and ESRD have also advanced atherosclerosis of cerebral vasculature and are at higher risk of ischemic stroke [11]. In the study by Nakayama et al. [12] decreased renal function was a significant risk factor for first symptomatic stroke event in general Japanese population where mortality from stroke is 3-fold higher than that in the United States. Furthermore, in several studies higher prevalence of renal dysfunction in patients with ischemic stroke was reported [13, 14]. Renal dysfunction was also a predictor of mortality in patients with stroke $[11,15]$. A meta-analysis of 21 studies showed that patients with renal dysfunction are at greater risk of future ischemic stroke [16]. Furthermore, Koren-Morag et al. [17] found that patients with established CHD and renal dysfunction are at higher risk of further ischemic stroke. Renal dysfunction was established as an important independent prognostic marker for ischemic stroke risk also after adjustment for traditional cerebrovascular risk factors in this study [17]. The mechanisms by which renal dysfunction increases CV risk are complex [15].

The possible impact of renal dysfunction on $\mathrm{CV}$ events after stroke is not well known. Tsagalis et al. [13] demonstrated that renal function on admission appears to be a significant independent prognostic factor for long term mortality and new CV morbidity over a 10 -year period.

The aim of our study was to define the impact of renal dysfunction on future $\mathrm{CV}$ events (including ACS, recurrent stroke, peripheral arterial disease $[\mathrm{PAD}]), \mathrm{CV}$ death and total mortality in patients suffering from ischemic stroke.

\section{Methods}

In our prospective study, all 390 Caucasian patients with ischemic stroke treated at our Department of Neurology in 1 year period were included. Patients were followed from the day of admission until their death or maximal 56 months (from 1 to 1673 days). Acute ischemic stroke was defined according to World Health Organization criteria [18]. The stroke was diagnosed in patients with appropriate clinical event and was confirmed with computed tomography. The patients with transitory ischemic attack (TIA) were excluded from the study. A study neurologist reviewed all cases. On admission, a quantitative measurement of neurologic deficit was performed according to the National Institutes of Health Stroke Scale (NIHSS1) [19]. The same measurement was performed at the day of discharge from the hospital (NIHSS2). We collected blood samples from all the patients. Serum creatinine, serum cholesterol (high density lipoprotein cholesterol [HDL-C] and low density lipoprotein cholesterol [LDL-C]), triglycerides, high sensitive $\mathrm{C}$-reactive protein (hsCRP) and serum albumin were measured by routine laboratory methods.

Diabetes mellitus was defined if the patient had already been treated for diabetes mellitus (information obtained by a questionnaire from patients and/ /or their relatives) or fasting glucose value during hospital treatment was higher than $7 \mathrm{mmol} / \mathrm{L}$ [20].

The arterial hypertension was defined as present if the patient had already been treated for hypertension (information obtained by a questionnaire from patients and/or their relatives) or the average measure blood pressure was $\geq 140 \mathrm{~mm} \mathrm{Hg}$ systolic or $\geq 90 \mathrm{~mm} \mathrm{Hg}$ diastolic based on 3 different measurements during hospitalization [21].

With questionnaire we also gathered data on smoking habits, patients were divided in two 
subgroups: smokers (present) and non-smokers. According to patient's weight and height the body mass index (BMI) was calculated using BMI formula: body weight (in kilograms)/stature (height, in meters) squared.

Estimated glomerular filtration rate (eGFR) was calculated according to the abbreviated Modification of Diet in Renal Disease (aMDRD) formula, which is standardized for a $1.73 \mathrm{~m}^{2}$ body surface area [7]. Renal dysfunction was defined as eGFR under $60 \mathrm{~mL} / \mathrm{min} / 1.73 \mathrm{~m}^{2}$.

The CV events were defined as documented ACS, documented recurrent ischemic stroke or documented PAD. Electrocardiography was recorded and strips were reviewed by a study cardiologist. Atrial fibrillation (AF) was documented.

Informed consent was obtained from each patient. The study protocol confirms to the ethical guidelines of the 1975 Declaration of Helsinki. The study was approved by National Medical Ethic Committee of the Republic of Slovenia.

\section{Statistical analysis}

SPSS for Windows (version 19.0.1) was used. Arithmetic mean values and standard deviation were calculated. Survival rates were analyzed using Kaplan-Meier survival curves. T-test and Mann-Whitney test were performed to compare data of patients who survived or died (total and CV mortality) and to compare data of patients with or without $\mathrm{CV}$ events. Cox regression model was used to access the influence of renal dysfunction on $\mathrm{CV}$ events, CV mortality or total mortality. The model was also adjusted for several observed cofactors: age, gender, smoking, diabetes, hypertension, $\mathrm{AF}$, HDL-C and LDL-C, triglycerides, serum albumin, hsCRP, NIHSS1 and NIHSS2 score.

\section{Results}

Of all included patients $183(46.9 \%)$ were women and $207(53.1 \%)$ were men. The mean age of the patients included was $70.9 \pm 11.64$ (ranged: 36-96) years. The mean eGFR was $66.97 \pm$ $\pm 21.45 \mathrm{~mL} / \mathrm{min} / 1.73 \mathrm{~m}^{2}$. In $153(39.2 \%)$ patients renal dysfunction (defined as eGFR under $60 \mathrm{~mL}$ / $/ \mathrm{min} / 1.73 \mathrm{~m}^{2}$ ) was found. In majority of patients ( $\mathrm{n}=135 ; 34.6 \%$ ) eGFR was between 60 and $30 \mathrm{~mL} /$ $/ \mathrm{min} / 1.73 \mathrm{~m}^{2}$ and in only $6(1.5 \%)$ patients eGFR was under $15 \mathrm{~mL} / \mathrm{min} / 1.73 \mathrm{~m}^{2}$. Basic characteristics of our patients are presented in Table 1 .

Our patients were followed from the day of admission to the hospital until maximal 1673 days or until their death. In the follow-up period in
Table 1. Basic characteristics of our patients.

\begin{tabular}{lc}
\hline Age [years] & $70.9 \pm 11.64$ \\
Hypertension (proportion) & $80.3 \%(313 / 390)$ \\
Smoking (proportion) & $15.6 \%(61 / 390)$ \\
Diabetes mellitus (proportion) & $25.6 \%(100 / 390)$ \\
Atrial fibrillation (proportion) & $20.8 \%(81 / 390)$ \\
eGFR [mL/min/1.73 m $^{2}$ ) & $66.97 \pm 21.45$ \\
Total cholesterol [mmol/L] & $5.16 \pm 1.30$ \\
LDL-C [mmol/L] & $3.18 \pm 1.08$ \\
HDL-C [mmol/L] & $1.22 \pm 0.34$ \\
Triglycerides [mmol/L] & $1.92 \pm 2.23$ \\
hsCRP [mg/L] & $17.8 \pm 35.91$ \\
Serum albumin [mmol/L] & $40.08 \pm 5.05$ \\
BMI [kg/m ${ }^{2}$ ] & $27.32 \pm 4.51$ \\
NIHSS1 [points] & $9.6 \pm 5.31$ \\
NIHSS2 [points] & $8.07 \pm 7.44$ \\
\hline
\end{tabular}

eGFR - estimated glomerular filtration rate; LDL-C — low density lipoprotein cholesterol; HDL-C - high density lipoprotein cholesterol; hsCRP - high sensitive C-reactive protein; BMI — body mass index; NIHSS1 - National Institutes of Health Stroke Scale - on admission; NIHSS2 - National Institutes of Health Stroke Scale - on discharge

$36(9.2 \%)$ patients ACS, in $102(26.2 \%)$ recurrent ischemic stroke and in $44(11.3 \%)$ PAD were documented. In $213(54.6 \%)$ patients no CV events were found; 191 (49\%) patients died, in 118 (30.3\%) patients CV death was confirmed.

The Kaplan-Meier survival analysis showed that total mortality ( $\mathrm{p}<0.003$ ) (Fig. $1 \mathrm{~A}$ ) and CV mortality $(\mathrm{p}<0.01)$ (Fig. 1B) were higher in patients with renal dysfunction.

The patients who died were older, had higher prevalence of renal dysfunction, more $\mathrm{AF}$, lower level of LDL-C and albumin, higher hsCRP, and the NIHSS score was higher on admission and on discharge. Similar data were found comparing patients who died due to CV events or survived. No statistically significant difference in presence of diabetes, hypertension, smoking, total and HDL-C, triglycerides and BMI were found (Table 2).

Comparing the groups of patients with or without CV events (using T-test and Mann-Whitney test) in observational period patients with $\mathrm{CV}$ events were older, they had more frequent diabetes and AF, NIHSS score was higher on admission and on discharge. No statistically significant differences in presence of hypertension, smoking habits, total cholesterol, LDL-C and HDL-C, triglycerides, hsCRP and albumin were found. No difference in the presence of renal dysfunction was found (Table 3).

According to Cox's regression analysis renal dysfunction was predictor of CV events, CV morta- 


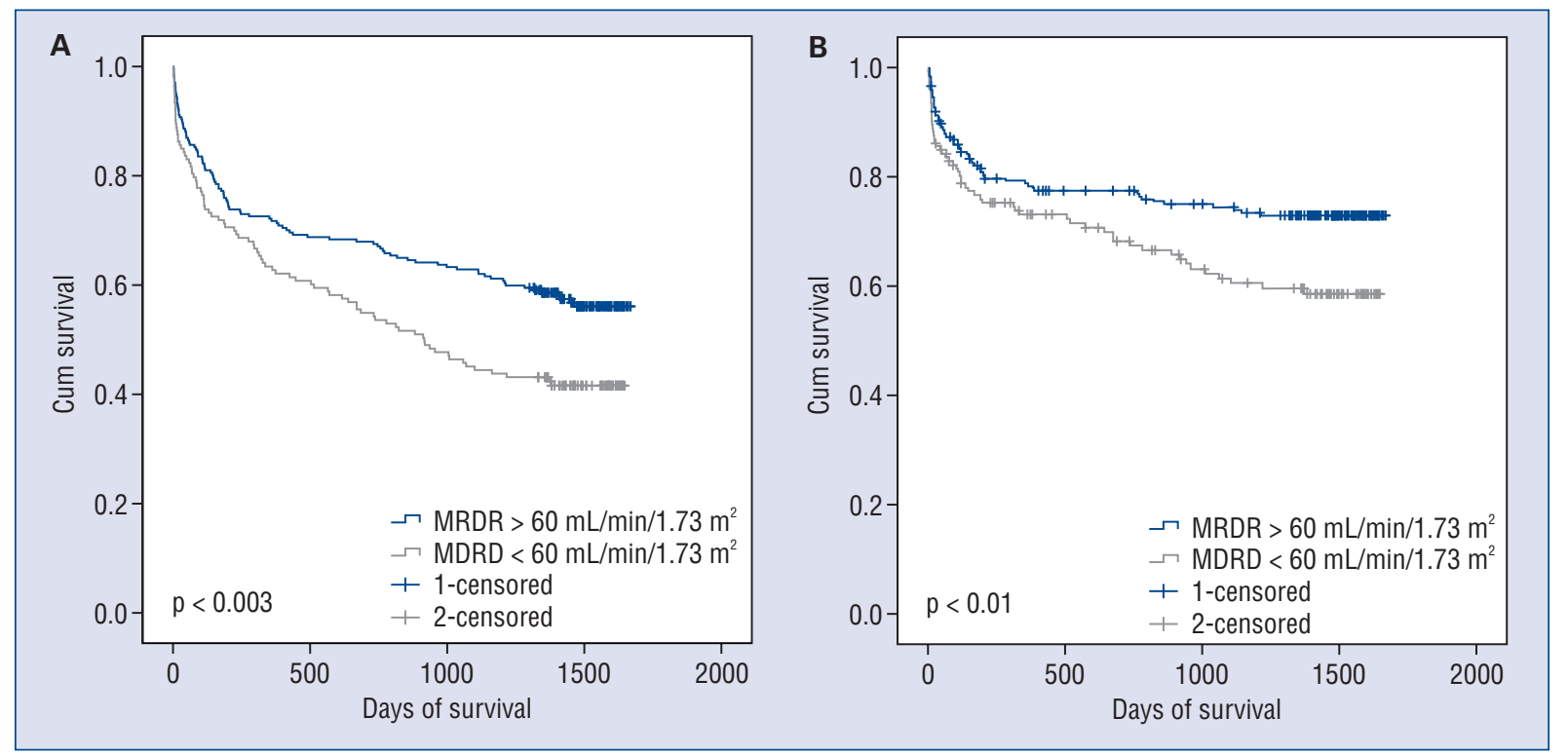

Figure 1. A. The Kaplan-Meier survival analysis of total mortality; B. The Kaplan-Meier survival analysis of cardiovascular mortality.

Table 2. Comparing patients who died (total and cardiovascular mortality) or survived.

\begin{tabular}{lccc} 
& Survived & \multicolumn{2}{c}{ Died } \\
\cline { 3 - 3 } & & Total mortality & Cardiovascular mortality \\
\hline Age [years] & $67.18 \pm 11.53$ & $74.93 \pm 10.4^{* *}$ & $75.37 \pm 9.96^{* *}$ \\
Hypertension (proportion) & $79.4 \%(158 / 199)$ & $81.2 \%(155 / 191)$ & $81.4 \%(96 / 118)$ \\
Smoking (proportion) & $18.1 \%(36 / 199)$ & $13.1 \%(25 / 191)$ & $11.0 \%(13 / 118)$ \\
Diabetes mellitus (proportion) & $24.1 \%(48 / 199)$ & $27.2 \%(52 / 191)$ & $31.4 \%(37 / 118)$ \\
Atrial fibrillation (proportion) & $14.1 \%(28 / 199)$ & $27.7 \%(53 / 191)^{*}$ & $29.7 \%(35 / 118)^{* *}$ \\
Total cholesterol [mmol/L] & $5.43 \pm 1.37$ & $4.88 \pm 1.16^{* *}$ & $4.93 \pm 1.19^{* *}$ \\
LDL-C [mmol/L] & $3.32 \pm 1.18$ & $3.02 \pm 0.96^{*}$ & $3.03 \pm 0.94^{*}$ \\
HDL-C [mmol/L] & $1.25 \pm 0.33$ & $1.19 \pm 0.36$ & $1.22 \pm 0.38$ \\
Triglycerides [mmol/L] & $2.11 \pm 2.57$ & $1.73 \pm 1.80$ & $1.65 \pm 1.49$ \\
hsCRP [mg/L] & $10.01 \pm 32.18$ & $25.67 \pm 37.88^{* *}$ & $26.26 \pm 34.26^{* *}$ \\
Serum albumin [mmol/L] & $41.86 \pm 3.99$ & $38.22 \pm 5.36^{* *}$ & $38.21 \pm 5.51^{* *}$ \\
BMI [kg/m ${ }^{2}$ ] & $27.84 \pm 4.43$ & $26.77 \pm 4.54^{*}$ & $27.00 \pm 4.38$ \\
NIHSS1 [points] & $7.78 \pm 3.92$ & $11.49 \pm 5.89^{* *}$ & $12.05 \pm 6.15^{* *}$ \\
NIHSS2 [points] & $4.36 \pm 3.17$ & $11.95 \pm 8.57^{* *}$ & $14.53 \pm 9.13^{* *}$ \\
Renal dysfunction (proportion) & $32.2 \%(64 / 199)$ & $46.6 \%(89 / 191)^{*}$ & $48.3 \%(57 / 118)^{* *}$ \\
\hline
\end{tabular}

*Statistically significant at level $\mathrm{p}<0.05$; ${ }^{*}$ Statistically significant at level $\mathrm{p}<0.001$; LDL-C - low density lipoprotein cholesterol; HDL-C — high density lipoprotein cholesterol; hsCRP - high sensitive C-reactive protein; BMI — body mass index; NIHSS1 — National Institutes of Health Stroke Scale — on admission; NIHSS2 - National Institutes of Health Stroke Scale — on discharge

lity and total mortality. Renal dysfunction remained a strong predictor of $\mathrm{CV}$ and total mortality also in adjusted Cox regression model (Table 4). The probability that patients after ischemic stroke with renal dysfunction will have $\mathrm{CV}$ event is 1.6 times higher than in patients without renal dysfunction. The probability for patients with renal dysfunction for total mortality is 1.9 times higher and for CV mortality 2.4 times higher than in patients without renal dysfunction. 
Table 3. Comparing data for patients with or without cardiovascular events.

\begin{tabular}{lcc}
\hline & \multicolumn{2}{c}{ Cardiovascular events } \\
\cline { 2 - 3 } & Without & With \\
\hline Age [years] & $68.92 \pm 12.43$ & $73.44 \pm 10.11^{* *}$ \\
Hypertension (proportion) & $80.3 \%(171 / 213)$ & $80.2 \%(142 / 177)$ \\
Smoking (proportion) & $17.8 \%(38 / 213)$ & $13 \%(23 / 177)$ \\
Diabetes mellitus (proportion) & $20.7 \%(44 / 213)$ & $31.6 \%(56 / 177)^{*}$ \\
Atrial fibrillation (proportion) & $16.9 \%(36 / 213)$ & $25.4 \%(45 / 177)^{*}$ \\
Total cholesterol [mmol/L] & $5.31 \pm 1.36$ & $4.99 \pm 1.18^{*}$ \\
LDL-C [mmol/L] & $3.28 \pm 1.16$ & $3.05 \pm 0.98^{*}$ \\
HDL-C [mmol/L] & $1.21 \pm 0.34$ & $1.233 \pm 0.35$ \\
Triglycerides [mmol/L] & $2.14 \pm 2.75$ & $1.67 \pm 1.34^{*}$ \\
hsCRP [mg/L]) & $14.95 \pm 39.68$ & $20.97 \pm 30.54^{* *}$ \\
Serum albumin [mmol/L] & $40.77 \pm 4.86$ & $39.24 \pm 5.16^{*}$ \\
BMI [kg/m ${ }^{2}$ ] & $27.54 \pm 4.66$ & $27.04 \pm 4.33$ \\
NIHSS1 [points] & $8.49 \pm 4.49$ & $10.81 \pm 5.94^{* *}$ \\
NIHSS2 [points] & $5.40 \pm 4.44$ & $11.29 \pm 8.93^{* *}$ \\
Renal dysfunction (proportion) & $36.2 \%(77 / 213)$ & $42.9 \%(76 / 177)$ \\
\hline
\end{tabular}

*Statistically significant at level $\mathrm{p}<0.05$; * Statistically significant at level $\mathrm{p}<0.001$; LDL-C - low density lipoprotein cholesterol; HDL-C — high density lipoprotein cholesterol; hsCRP - high sensitive C-reactive protein; BMI — body mass index; NIHSS1 — National Institutes of Health Stroke Scale — on admission; NIHSS2 - National Institutes of Health Stroke Scale — on discharge

Table 4. Renal dysfunction as a predictor of cardiovascular events, cardiovascular and total mortality.

\begin{tabular}{lllccc}
\hline & & B & p-value & HR & $95 \%$ Cl \\
\hline Cardiovascular events & NA & 0.321 & $<0.036$ & 1.379 & $1.022-1.861$ \\
& A & 0.468 & $<0.007$ & 1.597 & $1.139-2.238$ \\
Cardiovascular mortality & NA & 0.468 & $<0.011$ & 1.596 & $1.112-2.291$ \\
& A & 0.914 & $<0.0001$ & 2.494 & $1.633-3.807$ \\
Total mortality & NA & 0.422 & $<0.004$ & 1.525 & $1.147-2.027$ \\
& A & 0.637 & $<0.0001$ & 1.891 & $1.371-2.607$ \\
\hline
\end{tabular}

A — adjusted model; B — regression coefficient; $\mathrm{Cl}$ — confidence interval; HR — hazard ratio; NA — non adjusted model

\section{Discussion}

In our prospective study, 390 Caucasian adult patients with ischemic stroke were enrolled. The prevalence of renal dysfunction was $39.2 \%$ and was higher than in general adult population [2, 6, 22]. In only few studies the prevalence of renal dysfunction in patients with ischemic stroke was reported. In study by Tsagalis et al. [13] including patients with acute stroke (ischemic stroke and intra-cerebral hemorrhage), the prevalence of renal dysfunction was $28 \%$. Furthermore, the prevalence of renal dysfunction in patients with CVD is also higher than in general population, reported prevalence of renal dysfunction in patients with CHD is $18-37 \%$ [8, 10, 13, 23]. In study by Koren-Morag et al. [17], where patients with stable CVD were included, the prevalence of ischemic stroke or TIA was observed; the prevalence of renal dysfunction of included patients was $20 \%$. Further studies according to the prevalence of renal dysfunction in patients with ischemic stroke are still needed.

Patients with renal dysfunction are at increased risk of CVD and in some studies renal dysfunction was an independent risk factor for developing CVD including stroke $[8,12,16,23,24]$. In meta-analysis by Lee et al. [16], renal dysfunction defined as eGFR $<60 \mathrm{~mL} / \mathrm{min} / 1.73 \mathrm{~m}^{2}$ was independently related to incident ischemic and hemorrhagic stroke. Renal dysfunction was an independent predictor of the overall composite CV events during the follow-up in study by Tsagalis et al. [13]. The composite $\mathrm{CV}$ events in this study included recurrent stroke, new myocardial infarction or unstable angina, new 
onset of heart failure, sudden death with or without resuscitation, clinical onset of peripheral arteriopathy and thoracic or abdominal aortic rupture. More than $45 \%$ of our patients with ischemic stroke suffered at least one CV event in the observational period. The probability that patients with renal dysfunction will have CV event was 1.6 times higher than in patients without renal dysfunction.

Renal dysfunction predicted total mortality in some previous studies; in these studies subgroups of patients with ischemic or hemorrhagic stroke were not analyzed. Even less is known about CV mortality after ischemic stroke. In our study, in observational period 191 (49\%) patients died, among them 118 (30.3\%) due to CV events. Patients who died (total and CV mortality) had higher prevalence of renal dysfunction. The Kaplan-Meier survival analysis showed that total and CV mortality are higher in patients with renal dysfunction after ischemic stroke. Renal dysfunction was also a powerful predictor of total and CV mortality in patients with ischemic stroke according to Cox's regression model.

The associations between renal dysfunction and CVD including ischemic stroke are numerous. Traditional and nontraditional risk factors (age, gender, hypertension, diabetes, dyslipidemia, smoking, hsCRP, etc.) may play important roles [25]. Our patients who died (total and CV mortality) were older compared to the survivors. Patients who suffered new CV events in the follow-up period were also older. Age was associated with worse outcome also in the studies of MacWalter et al. [15] and Tsagalis et al. [13]. Age was an independent predictor of composite CV events in study by Tsagalis et al. [13]. This is important because in the developed countries population is quickly aging.

Hypertension, diabetes and smoking are known important risk factors for stroke. In one previous study, patients with documented CHD and renal dysfunction had baseline higher proportion of hypertension, were less likely to smoke and there was no difference in presence of diabetes compared to the patients without renal dysfunction [17]. However, the impact of hypertension, diabetes and smoking on prognosis or further $\mathrm{CV}$ events after ischemic stroke is not known. In our study, no differences between patients who died (total and CV mortality) or survived were found in presence of hypertension, diabetes or smoking. No difference in hypertension and smoking was found also comparing patients with and without $\mathrm{CV}$ events. The prevalence of diabetes was higher in patients with $\mathrm{CV}$ events. No difference in BMI was found in our patients.
$\mathrm{AF}$ is a powerful risk factor for ischemic stroke, it is common asymptomatic and therefore underestimated $[9,26]$. Patients with $\mathrm{AF}$ are more likely to suffer a recurrent stroke [26]. Also in our study the prevalence of AF was higher in patients according to total mortality and in patients who died due to $\mathrm{CV}$ events or had nonfatal $\mathrm{CV}$ events. $\mathrm{AF}$ was a predictor of $\mathrm{CV}$ events also in the study by Tsagalis et al. [13].

Dyslipidemia is a traditional risk factor for CVD [2, 4]. In our study, surprisingly no difference in level of LDL-C, HDL-C or level of triglycerides between patients after ischemic stroke with or without CV events was found. Patients who died (total mortality and CV mortality) had lower LDL-C. Conflicting results on the role of dyslipidemia and incident stroke, also in studies including patients with renal dysfunction, are reported. In the study by Tsagalis et al. [13] no difference in dyslipidemia in patients after stroke and renal dysfunction was found, but in the study by Koren-Morag et al. [17] higher level of HDL-C and lower level of triglycerides in patients with known CHD and renal dysfunction after ischemic stroke were found.

In Atherosclerosis Risk in Communities (ARIC) study baseline characteristics showed that patients with renal dysfunction had higher HDL-C and there was no difference in LDL-C [27]. More studies are needed to define the impact of dyslipidemia in patients with ischemic stroke and renal dysfunction.

High serum CRP has been related to the risk of cerebrovascular and $\mathrm{CV}$ events in patients with and without renal dysfunction and was associated with increased hazard for ischemic stroke in patients with known CVD [28]. No large study has prospectively assessed the value of CRP for prognostic short-term and long-term stratification of patients with ischemic stroke. Our patients who died (total and CV mortality) had higher hsCRP values. Patients who died had also lower serum albumin. Together with higher hsCRP values and lower LDL-C values possible association between malnutrition, inflammation and atherosclerosis is suggested, what is common in patients with terminal chronic renal failure and is strongly associated with accelerated atherosclerosis [29]. In our study, no differences in hsCRP and serum albumin in patients with or without $\mathrm{CV}$ events were found.

It is otherwise known that NIHSS has established reliability and validity for use in prospective clinical research, and predictive validity for short-term and long-term stroke outcome [30]. It is not surprising that our patients who died had 
higher NIHSS on admission and discharge and that higher NIHSS scores in patients with CV events were present.

\section{Limitations of the study}

The main limitation of our study is that it was done only in one medical center. Therefore, this fact limits the study's applicability to general population.

\section{Conclusions}

Our study demonstrated that the patients with ischemic stroke and renal dysfunction are at higher risk of long term $\mathrm{CV}$ and total mortality. The patients with ischemic stroke and renal dysfunction are also at higher risk of new CV morbidity.

Renal dysfunction should be added to other known prognostic factors in patients with ischemic stroke. Our results also emphasize the importance of identification and management of renal dysfunction in stroke patients.

\section{Conflict of interest: none declared}

\section{References}

1. Bhatnagar P, Scarborough P, Smeeton NC, Allender S. The incidence of all stroke and stroke subtype in the United Kingdom, 1985 to 2008: A systematic review. BMC Public Health, 2010; 10: 539.

2. Levey AS, Coresh J, Balk E et al. National Kidney Foundation. National Kidney Foundation practice guidelines for chronic kidney disease: Evaluation, classification, and stratification. Ann Intern Med, 2003; 139: 137-147.

3. Culleton BF, Larson MG, Evans JC et al. Prevalence and correlates of elevated serum creatinine levels: the Framingham Heart Study. Arch Intern Med, 1999; 159: 1785-1790.

4. Foley RN, Murray AM, Li S et al. Chronic kidney disease and the risk for cardiovascular disease, renal replacement, and death in the United States Medicare population, 1998 to 1999. J Am Soc Nephrol, 2005; 16: 489-495.

5. Muntner P, Hamm LL, Kusek JW, Chen J, Whelton PK, He J. The prevalence of nontraditional risk factors for coronary heart disease in patients with chronic kidney disease. Ann Intern Med, 2004; 140: 9-17.

6. National Kidney Foundation. K/DOQI clinical practice guidelines for chronic kidney disease: Evaluation, classification, and stratification. Am J Kidney Dis, 2002; 39: S1-S266.

7. Hojs R., Hojs-Fabjan T, Pecovnik-Balon B. Atherosclerosis in patients with end stage renal failure prior to initiation of hemodialysis. Renal Failure, 2003; 25: 247-254.

8. Bevc S, Penko M, Kanic V, Hojs R. Mortality of patients with renal dysfunction after percutaneous coronary intervention. Angiology, 2010; 61: 24-30.

9. Roger VL, Go AS, Lloyd-Jones DM et al. Heart Disease and Stroke Statistics--2011 Update: A Report from the American Heart Association. Circulation, 2011; 123: 18-209.

10. Sadeghi HM, Stone GW, Grines CL et al. Impact of renal insufficiency in patients undergoing primary angioplasty for acute myocardial infarction. Circulation, 2003; 108: 2769-2075.

11. Seliger SL, Gillen DL, Tirschwell D, Wasse H, Kestenbaum BR, Stehman-Breen CO. Risk factors for incident stroke among patients with end-stage renal disease. J Am Soc Nephrol, 2003; 14: 2623-2631.
12. Nakayama M, Metoki $\mathrm{H}$, Terawaki $\mathrm{H}$ et al. Kidney dysfunction as a risk factor for first symptomatic stroke events in a general Japanese population: The Ohasama study. Nephrol Dial Transplant, 2007; 22: 1910-1915.

13. Tsagalis G, Akrivos T, Manios E, Stamatellopoulos K, Laggouranis A, Vemmos KN. Renal dysfunction in acute stroke: An independent predictor of long-term all combined vascular events and overal mortality. Nephrol Dial Transplant, 2009; 24: 194-200.

14. Hojs Fabjan T, Hojs R, Tetickovic E, Pecovnik Balon B. Ischaemic stroke: Impact of renal dysfunction on in-hospital mortality. Eur J Neurol, 2007; 14: 1351-1356.

15. MacWalter RS, Wong SY, Wong KY et al. Does renal dysfunction predict mortality after acute stroke? A 7-year follow-up study. Stroke, 2002; 33: 1630-1635.

16. Lee M, Saver JL, Chang KH, Liao HW, Chang SC, Ovbigale B. Low glomerular filtration rate and risk of stroke: Meta-analysis. BMJ, 2010; 341: c4249.

17. Koren-Morag N, Goldbourt U, Tanne D. Renal dysfunction and risk of ischemic stroke or TIA in patients with cardiovascular disease. Neurology, 2006; 67: 224-228.

18. Thorvaldsen P; Asplund K; Kuulasmaa K., Rajakangas AM, Schroll M; for the WHO MONICA Project. Stroke Incidence, Case Fatality, and Mortality in the WHO MONICA Project. Stroke, 1995; 26: 361-367.

19. Muir KW, Weir CJ, Murray GD, Povey C, Lees KR. Comparison of Neurological Scales and Scoring Systems for Acute Stroke Prognosis. Stroke, 1996; 27: 1817-1820.

20. Rydén L, Standl E, Bartnik M et al. Guidelines on diabetes, pre-diabetes, and cardiovascular diseases: executive summary. The Task Force on Diabetes and Cardiovascular Diseases of the European Society of Cardiology (ESC) and of the European Association for the Study of Diabetes (EASD). Eur Heart J, 2007; 28: 88-136.

21. Mancia G, De Backer G, Dominiczak A et al. 2007 Guidelines for the management of arterial hypertension: The Task Force for the Management of Arterial Hypertension of the European Society of Hypertension (ESH) and of the European Society of Cardiology (ESC). Eur Heart J, 2007; 28: 1462-1536.

22. Coresh J, Astor BC, Greene T, Eknoyan G, Levey AS. Prevalence of chronic kidney disease and decreased kidney function in the adult US population: Third National Health and Nutrition Examination Survey. Am J Kidney Dis, 2003; 41: 1-12.

23. Penko M, Bevc S, Kanič V, Hojs R. The prevalence of chronic kidney disease in patients with acute coronary syndrome. Zdrav Vestn, 2007; 76: 151-157.

24. Go AS, Chertow GM, Fan D, Mc Culloch CE, Hsu CY. Chronic kidney disease and the risks of death, cardiovascular events, and hospitalization. N Engl J Med, 2004; 351: 1296-1305.

25. Muntner P, He J, Astor BC, Folsom AR, Coresh J. Traditional and nontraditional risk factors predict coronary heart disease in chronic kidney disease: Results from the atherosclerosis risk in communities study. J Am Soc Nephrol, 2005; 16: 529-538.

26. Camm AJ, Kirchhof P, Lip GY et al.; European Heart Rhythm Association; European Association for Cardio-Thoracic Surgery. Guidelines for the management of atrial fibrillation. The Task Force for the Management of Atrial Fibrillation of the European Society of Cardiology (ESC). Eur Heart J, 2010; 31: 2369-2429.

27. Abramson JL, Jurkovitz CT, Vaccarino V, Weintraub WS, McClellan W. Chronic kidney disease, anemia, and incident stroke in a middle-aged, community-based population: The ARIC study. Kidney Int, 2003; 64: 610-615.

28. Tanne D, Benderly M, Goldbourt U et al. C-reactive protein as a predictor of incident ischemic stroke among patients with preexisting cardiovascular disease. Stroke, 2006; 37: 1720-1724.

29. Stenvinkel P, Heimburger O, Paultre F et al. Strong association between malnutrition, inflammation and atherosclerosis in chronic renal failure. Kidney Int, 1999; 55: 1899-1911.

30. Hojs Fabjan T. Predicting short-term (30-day) mortality in patients with ischemic stroke using the baseline score of the National Institutes of Health Stroke Scale. Acta Medico-Biotechnica, 2012; 5: 46-53. 